\title{
Pergolide reduced involuntary periodic leg movements and improved sleep in the restless legs syndrome
}

Wetter TC, Stiasny K, Winkelmann J, et al. A randomized controlled study of pergolide in patients with restless legs syndrome. Neurology 1999 Mar;52:944-50.

QUESTION: In patients with idiopathic restless legs syndrome (RLS), is pergolide effective for improving symptoms and sleep?

\section{Design}

12-week randomised (allocation concealed*), doubleblind, placebo-controlled crossover trial.

\section{Setting}

Outpatient clinic for movement and sleep disorders in Germany.

\section{Patients}

Patients were 28-70 years of age and had an irresistible urge to move that was related to sensory symptoms of the lower limbs; motor restlessness; worsening of symptoms at rest with some relief found through activity; increased symptom severity in the evening or at night; abnormal periodic leg movement (PLM) index; sleep-onset latency $>25$ minutes or sleep efficiency $<85 \%$; and normal values for serum chemistry and urinalysis. Exclusion criteria were secondary RLS, other primary sleep disorders, history of substance abuse, pregnancy, lactation, hypersensitivity to ergot derivatives, current antihistamine use, serious medical conditions, or current diagnosis or history of malignant melanoma. 30 patients were enrolled, and 28 (93\%, mean age 57 y, 57\% women) were studied.

\section{Intervention}

Patients were allocated to pergolide, $0.05 \mathrm{mg}(\mathrm{n}=15)$, or placebo $(n=15)$. Doses given once daily were titrated to best improvement over 2 weeks (maximum $0.75 \mathrm{mg} / \mathrm{d}$ ) and held constant for a further 2 weeks. After 1 week of down titration and a 1-week washout, patients crossed over to placebo $(n=14)$ or pergolide $(n=14)$. The mean plateau dose of pergolide was $0.51 \mathrm{mg}$ (range $0.25-0.75$ $\mathrm{mg}$ ). All patients received domperidone, $20 \mathrm{mg} 3$ times/day, to prevent peripheral dopaminergic side effects.

\section{Main outcome measures}

PLM index (number of PLMs/h) during sleep and wakefulness and total sleep time (assessed by polysomnography); subjective sleep quality (scores 1 [bad] to 5 [good]); and adverse events.

\section{Main results}

The PLM index was lower in the pergolide group than in the placebo group during sleep and wakefulness (mean PLM index 5.7 v 55, p <0.001) and wakefulness alone (mean PLM index 17 v 70, $\mathrm{p}<0.001$ ). Pergolide led to less sleep arousal than did placebo (mean arousal index for PLMs during sleep $2.2 v 32.1, \mathrm{p}<0.001)$. Pergolide also led to longer total sleep time (mean sleep time $373.6 v 261.9 \mathrm{~min}, \mathrm{p}<0.001)$ and greater subjective quality of sleep (mean score $3.0 v 2.2, \mathrm{p}<0.001$ ) than placebo. 5 patients receiving pergolide and 2 patients receiving placebo had severe adverse events; 1 patient withdrew because of severe abdominal pain during placebo treatment.

\section{Conclusion}

In patients with the restless legs syndrome, pergolide reduced involuntary periodic leg movements, increased the amount of sleep, and improved subjective sleep quality.

*See glossary.

\section{COMMENTARY}

RLS is common (2-5\% of the population) and distressing, especially when it occurs at night. The mean age of onset is 25-30 years. It is a lifelong condition and is becoming increasingly common in elderly people. At least one third of cases are hereditary and are often autosomal dominant. 80\% of people with RLS have PLMs in sleep (5\% of people $30-50$ $\mathrm{y}$ and $29 \%$ of people $50-60 \mathrm{y}$ ) in the general population. ${ }^{1} \mathrm{Up}$ to $19 \%$ of women experience RLS during pregnancy, but it is usually temporary. ${ }^{1}$

RLS must be distinguished from akathisia, myoclonus, pain from lumbosacral spinal stenosis, or "painful feet and moving toes." The pathophysiology of RLS is unclear. RLS may accompany iron or vitamin deficiency, uremia, occlusive arterial disease, congestive cardiac failure, peripheral neuropathy, and other neurological diseases and may respond to treatment of the primary disorder. Diagnosis of RLS can usually be made clinically, and sleep studies are not essential. Sleep studies, however, provide useful objective outcome measures for research ${ }^{2}$ and treatment trials, which are plagued by prominent placebo responses. ${ }^{1}$

The best validated treatment option is the use of dopaminergic drugs. Bedtime levodopa provides rapid improvement, ${ }^{1}$ but rebound symptoms may occur later at night. Benzodiazepines, especially clonazepam, may improve sleep quality, and opioids reduce sleep arousals. ${ }^{1}$. Both drug classes, however, have risk for dependence. Randomised controlled trials of carbamazepine treatment have shown promise, and clonidine, bromocriptine, and baclofen may also be helpful. ${ }^{1}$

This well-done study by Wetter and colleagues supports the results of open studies ${ }^{3}$ reporting that pergolide is effective in the short term and is thus a reasonable treatment option. It should now be tested against other available treatments for comparative efficacy. Until such comparisons are made, levodopa and clonazepam are less expensive treatments that can work well and should probably be tried first.

A Peter Moore, MB, ChB, MD Walton Centre for Neurology and Neurosurgery Liverpool, UK

1 O'Keeffe ST. Arch Intern Med 1996;156:243-8.

2 Montplaisir J, Boucher S, Poirier G, et al. Mov Disord 1997; 12:61-5.

3 Winkelmann J, Wetter TC, Stiasny K, et al. Mov Disord 1998;13:566-9. 